\title{
Serum miR-21 may be a Potential Diagnostic Biomarker for Diabetic Nephropathy
}

\author{
Authors \\ J. Wang ${ }^{1 *}$, L. Duan ${ }^{2 *}$, L. Tian ${ }^{1}$, J. Liu' ${ }^{1}$, S. Wang ${ }^{3}$, Y. Gao ${ }^{4}$, J. Yang ${ }^{5}$ \\ Affiliations \\ Affiliation addresses are listed at the end of the article
}

\author{
Key words \\ - serum miR-21 \\ - diabetic nephropathy \\ - structure and function \\ - diagnostic biomarker
}

\begin{abstract}
$\nabla$

MiRNAs play important roles in initiation and progress of many pathologic processes. MiR-21 was closely associated with diabetic nephropathy (DN). However, whether serum miR-21 was as a potential diagnostic biomarker for DN and the relationship between serum miR-21 and tissue miR-21 remained unclear. In this study, real-time RT-PCR, cell transfection, luciferase reporter gene assays, western blot and confocal microscope were used, respectively. Here, we found that serum and renal tissue miR-21 was significantly elevated with the progress of DN. Moreover, luciferase reporter gene assays showed that

\begin{tabular}{ll}
\hline \multicolumn{2}{l}{ Abbreviations } \\
$\nabla$ & \\
DN & Diabetic nephropathy \\
ACR & urine albumin creatinine ratio \\
Ccr & creatinine clearance ratio \\
AntagomiR-21 & miR-21 antagonist \\
GBM & glomerular basement membrane \\
GA & glomerular area \\
CCF & content of collagen fibers
\end{tabular}
\end{abstract}

\section{Introduction}

$\nabla$

Diabetic nephropathy (DN) is a chronic, progressive process that ultimately leads to renal fibrosis and end-stage renal failure, a devastating disorder that requires dialysis or kidney transplantation [1-3]. KK-Ay mouse was considered suitable as a polygenic model for human type- 2 diabetes mellitus and was produced by transferring the yellow obese gene ( $\mathrm{A}^{\mathrm{y}}$ allele) into the $\mathrm{KK}-\mathrm{Ay} / \mathrm{Ta}$ mice. Renal lesions in KK-Ay mice closely resem-

\footnotetext{
* Jinyang Wang and Lijun Duan contributed equally to this study.
}

smad7 was a validated miR-21 target, cell transfection showed that miR-21 overexpression downregulated target smad7 expression. Interestingly, serum miR-21 was significantly consistent with the alterations of tissue miR- 21 with the development of DN. In addition, serum miR-21 was also positively correlated with GBM, GA, ACR and CCF, while negatively correlated with Ccr. Importantly, antagomiR-21 not only alleviated GBM, GA, ACR and CCF, but also ameliorated Ccr by increasing target smad7. In conclusion, our data demonstrated that serum miR-21 was closely associated with renal structure and function, and serum miR-21 may be regarded as a potential diagnostic biomarker of DN.

bled human DN, which develop marked high glucose, albuminuria and renal fibrosis [4].

MicroRNAs (miRs) are endogenous non-coding small RNA, 20-22 nucleotides in length, which bind to the 3'-UTR of target genes, thereby, repress translation and/or induce degradation of target gene mRNAs [5]. Increasing evidences have indicated that miRNAs play significant roles in many diseases including diabetes mellitus and its complications [6]. For example, miR-29 aggravates insulin resistance and inhibits fibrosis $[7,8]$. miR-377 can lead to increased fibronectin production in DN [9]. miR-451 regulates p38 MAPK signaling by targeting of Ywhaz and suppresses the mesangial hypertrophy in early DN [10]. miR503 contributes to diabetes mellitus-induced impairment of endothelial function and reparative angiogenesis after limb ischemia [11]. miR192 may be a critical downstream mediator of TGF- $\beta /$ Smad3 signaling in the development of renal fibrosis [12]. Recent reports have documented that miR-21 plays a crucial role in DN and kidney fibrotic diseases [13,14]. For example, miR-21 expression was increased in kidney biopsies from diabetic patients and DN mice [15]. miR-21 expression increases rapidly in cultured 
murine podocytes after exposure to TGF- $\beta 1$ and is higher in kidneys of TGF- $\beta 1$-transgenic mice than wild-type mice [16]. MiR21 orchestrates high glucose-induced signals to TOR complex-1, resulting in renal cell pathology in diabetes [17]. miR-21 promotes fibrosis of the kidney by silencing metabolic pathways [18]. miR-21 protects from mesangial cell proliferation induced by $\mathrm{DN}$ in $\mathrm{db} / \mathrm{db}$ mice [19]. Interestingly, recent studies have shown that miR-21 is a key therapeutic target for DN and renal fibrosis $[14,20]$. Increased circulating miR-21 levels are associated with kidney fibrosis [21]. More importantly, our previous experiments showed that miR-21 was extensively distrubuted in renal tubular epithilial cells of cortical kidneys in DN mice, miR-21 overexpression promoted renal fibrosis by enhancing TGF- $\beta 1$-induced EMT [22,23]. However, whether serum miR-21 was as a potential diagnostic biomarker for DN and relationship between serum miR-21 and tissue miR-21 in DN remained unclear.

The aim of this study was to identify the relationship between serum miR-21 and tissue miR-21, and to explore whether serum miR-21 was a novel diagnostic biomarker of DN. The results suggest that both serum miR-21 was closely associated with renal structure and function, and serum miR-21 may be as a novel diagnostic biomarker for DN.

\section{Materials and Methods}

$\nabla$

\section{Animals and diabetic nephropathy models}

Male C57BL/6J (12 weeks of age, 24 mice) and KK-Ay mice (12 weeks of age, 48 mice) from Chinese Academy of Medical Sciences (Beijing, China) were individually housed in plastic cages with free access to food and water throughout the experiment. All mice were maintained in the same room under conventional conditions with a regular 12-h light/dark cycle with the temperature controlled at $24^{\circ} \mathrm{C} \pm 1^{\circ} \mathrm{C}$. To induce $\mathrm{DN}, \mathrm{C} 57 \mathrm{BL} / 6 \mathrm{~J}$ mice were fed by common forage ( $12 \%$ fat, $60 \%$ carbohydrate, and $28 \%$ protein), KK-Ay mice received research diets ( $58 \%$ fat, $25.6 \%$ carbohydrate, and $16.4 \%$ protein) for 4 weeks, random blood glucose (RBG) was checked by a portable glucometer from tail vein of each animal, C57BL/6J mice were classified as normal control group (NC group, $n=20$ ), KK-Ay mice were considered DN when their RBG was $\geq 300 \mathrm{mg} / \mathrm{dl}(16.7 \mathrm{mmol} / \mathrm{l})$ and ACR (urine albumin creatine ratio) was $\geq 300 \mathrm{ug} / \mathrm{mg}$ were detected, KK-Ay mice were considered as DN model suitable for human type-2 diabetes mellitus [4]. KK-Ay mice were randomly divided into DN model group (DN group, $n=24$ ), which were injected intraperitoneally with non-targeting negative control sequences and DN with antagomiR-21 treatment group (antagomiR-21 treatment group, $n=24$ ), which were injected intraperitoneally with antagomiR-21 (the antagonist of miR-21, 30mg/kg/d, Ribobio, China) for 8 weeks. Antagomir is a miRNA antagonist, which binds with mature miRNA in the body. Antagomir prevents miRNA and its target gene mRNA complementary pairing, and restrains the action of miRNA [24]. Renal tissue and serum samples were derived from 5 mice at every 4 weeks in each group for later use. Renal tissue from each mouse for western blot, RT-PCR, Masson, Picro-sirius red, electron microscope and light microscopy, respectively. Experiment protocol was approved by the Institutional Animal Care and Use Committee.

\section{Biochemical assays}

Serum creatinine (SCr) and body weight was measured at 12,16 , 20 and 24 weeks of age. Urinary albumin and creatinine were measured by immunoassay (DCA 2000 system, Germany). Urinary albumin creatinine ratio (ACR) was calculated as: $A C R=$ urinary albumin $(\mu \mathrm{g}) /$ urinary creatinine $(\mathrm{mg})$. Creatinine clearance (Ccr) ratio was calculated using the following equation [1]: $\mathrm{Ccr}$ $\left(\mathrm{mL} \mathrm{min}{ }^{-1} \mathrm{~kg}^{-1}\right)=$ [urinary $\mathrm{Cr}\left(\mathrm{mg} \mathrm{dL}^{-1}\right) \times$ urinary volume $(\mathrm{mL}) /$ serum $\left.\mathrm{Cr}\left(\mathrm{mg} \mathrm{dL}^{-1}\right)\right] \times[1000 /$ body weight $(\mathrm{g})] \times[1 / 1440(\mathrm{~min})]$ [25].

\section{Light and electron microscopy}

Tissue for light microscopy was fixed in 10\% phosphate-buffered formalin and then embedded in paraffin. 4-micrometer-thick sections were processed for hematoxylin-eosin staining by light microscopy. Tissues for electron microscope were fixed with $2 \%$ glutaraldehyde in $0.1 \mathrm{~mol} / \mathrm{L}$ phosphate buffer at $4{ }^{\circ} \mathrm{C}$ for $120 \mathrm{~min}$. Ultrathin sections were collected on 100-mesh copper grids and double stained with $4 \%$ uranyl acetate and lead citrate. The sections were examined with a Hitachi 7100 transmission electron microscope. Morphologic analyses were performed by an experienced pathologist who was blinded to the source of the tissue.

\section{Transfection of cultured HKCs}

To investigate the role of miR-21 in normal human kidney tubular epithelial cells (HKCs), obtained from Chinese Type Culture Collection (CTCC), we performed miR-21 transfection experiments, and cells were seeded at a density of $2 \times 10^{4}$ cells $/ \mathrm{cm}^{2}$ in serum-free DMEM/F12. In this study, cells were divided into the following groups: cells without transfection were used as blank control group. Cells transfected with miR-control lentivirus vector were used as miR-control group. Cells transfected with miR21 over-expression (pre-miR-21) lentivirus vector were used as miR-21 over-expression group (pre-miR-21 group). Cells transfected with miR-21 inhibitor lentivirus vector were used as miR21 inhibitor group. After $12 \mathrm{~h}$ transfection, the medium was changed and the HKCs were incubated with fresh serum-containing medium for another $48 \mathrm{~h}$. In our experiment, the most appropriate multiplicity of infection (MOI) for HKCs equals to 30 , all the transfected cells were measured and sorted $48 \mathrm{~h}$ later according to the green fluorescent protein (GFP) intensity by flow cytometry, and the transfection efficiency was above $97 \%$. The entire abovementioned lentivirus vector was custom-synthesized by Shanghai Genechem Co., Ltd, China. After 3 days of culturing, cells were harvested for RNA or protein isolation.

\section{Luciferase reporter gene assays}

To examine whether miR-21 regulates the expression of smad7, we transiently transfected miR-control plasmid, wild-type or mutant luciferase-smad7-3'UTR reporter) and miR-21 overexpressing plasmid into $40-50 \%$ confluent T293 cells (Genechem, Shanghai, China), which grown in a 24 -well plate. The cells were harvested $48 \mathrm{~h}$ after transfection, and luciferase activity was measured with a dual luciferase reporter assay kit (Promega, Madison, WI, USA) on a luminometer (Lumat LB9507).

\section{Real-time RT-PCR analysis}

For analysis of serum and renal tissue miR-21 expression, TaqMan miRNA assays (Applied Biosystems, California, and USA) were used for quantitative determination of miR-21 expression according to the manufacturer's instructions. The relative expression was normalized to the expression of U6 RNA (Applied 
Biosystems). Relative fold changes of gene expression were calculated by the $C T$ method and the values are expressed as $2^{-\Delta \Delta} \mathrm{Ct}$. All Real-time RT-PCRs were performed in duplicate.

\section{Western blot analysis}

Renal tissues were lysed in RIPA buffer with protease inhibitors (Roche). Protein concentrations were determined by bicinchoninic assay (Pierce Biotechnology, Rockford, IL, USA). Proteins were separated on $10 \%$ SDS-PAGE gels under reducing conditions and transferred to polyvinylidene difluoride membranes. The membranes was performed with rabbit polyclonal to col-IV antibody (Abcam) and polyclonal to Col-I antibody (Abcam), and then with secondary antibodies (1:5000, Roche), Membranes were stripped and reprobed with $\beta$-actin antibody (Sigma) and secondary antibody for data normalization.

\section{Statistical analysis}

Statistical analyses were performed by one-way ANOVA followed by the Bonferroni multiple comparison test (for comparison of more than 2 groups) or Student $t$ test (for comparison of 2 groups) and correlated analysis by Pearson's correlation test. A probability value of $<0.05$ was considered significant.

\section{Results}

\section{Relationship between serum miR-21 and tissue miR-21}

To explore the relationship between serum miR-21 and renal tissue miR-21, we measured serum miR-21 and renal tissue miR21 by real-time quantitative RT-PCR at different weeks of age. At 12 weeks of age, serum miR-21 was initially increased $(p<0.05)$. With the progress of DN, serum miR-21 level was further elevated until 24 weeks of age ( $\odot$ Fig. 1a. $p<0.05$ ). Interestingly, the alterations of serum miR-21 were significantly consistent with the expressions of renal tissue miR-21( $\bullet$ Fig. $\mathbf{1 b}, \mathbf{c} \mathrm{p}<0.05$ ). More importantly, serum miR-21 was positively correlated with tissue miR-21 expression by Pearson correlation analysis ( $\odot$ Fig. 1d, $\mathrm{r}=0.894, \mathrm{p}<0.05$ ). Next, to clarify whether antagomiR-21 decreased the expressions of serum and tissue miR-21, KK-Ay DN mice were injected intraperitoneally with antagomiR-21, we found that serum and tissue miR-21 were significantly decreased in antagomiR-21 group compared with DN group ( 0 Fig. 1a, b $\mathrm{p}<0.05$ ). Taken together, our results suggested that circulating serum miR-21 may reflect the changes of tissue miR-21.

\section{Relationship between serum miR-21 and renal function}

To evaluate the relationship between serum miR-21 and ACR/ $\mathrm{Ccr}$, and to further elucidate whether serum miR-21 could be as a novel diagnostic biomarker for DN, the changes of ACR and $\mathrm{Ccr}$ were examined. With the progress of DN (from 16 to 24 weeks of age), ACR began to increase and Ccr began to decline ( 0 Fig. 2a, b). Additionally, antagomiR-21 was able to significantly decrease ACR and increase $\mathrm{Ccr}(\boldsymbol{\otimes}$ Fig. 2a, b p $<0.05$ ). Interestingly, serum miR-21 expression was positively correlated with ACR ( $\odot$ Fig. 2c, $\mathrm{r}=0.970, \mathrm{P}=0.006)$ and negatively correlated with $\mathrm{Ccr}(\Theta \mathrm{Fig} . \mathbf{2 d}$, $r=-0.950, P<0.01)$. Importantly, we found that antagomiR-21 can decrease the levels of ACR and increase the levels of $\mathrm{Ccr}$ ( $\odot$ Fig. 2a, b, p < 0.05), Thus, our results suggested that serum miR-21 was closely associated with the markers of renal function (ACR and CCr), and that serum miR-21 may be as a novel diagnostic biomarker for DN.

\section{Relationship between serum miR-21 and morphological changes}

Numerous studies demonstrated that key characteristics of DN are characterized by glomerular basement membrane (GBM) thickened and mesangial matrix hyperplasia [1]. Here, to evaluate the relationship between serum miR-21 and morphological changes at 24 weeks of age, renal morphology was observed by light and electronic microscopy (EM) ( $\odot$ Fig. 3a). We found that GBM is normal and glomerular foot process is slender and tidy in control group. In contrast, GBM $(145 \pm 20.4$ vs. $100 \pm 18.6 \mathrm{~nm})$ thickened, foot process partly fused and structure disordered in DN group. Furthermore, compared with NC group, glomerular area (GA, $51.2 \pm 2.4$ vs. $32.9 \pm 3.6 \mathrm{um}^{2}, \mathrm{P}<0.05$ ) increased, GBM $(145 \pm 20.4$ vs. $100 \pm 18.6 \mathrm{~nm}, \mathrm{P}<0.05)$ thickened in DN group. Interestingly, the level of serum miR-21 was positively correlated with GBM and GA (Pearson correlation, $\mathrm{r}_{\mathrm{GBM}}=0.770$, $\left.\mathrm{r}_{\mathrm{GA}}=0.630, \mathrm{P}<0.05\right)$. Importantly, antagomiR-21 decreased GBM and GA ( $\odot$ Fig. $3 c$, d). Taken together, the results suggested that

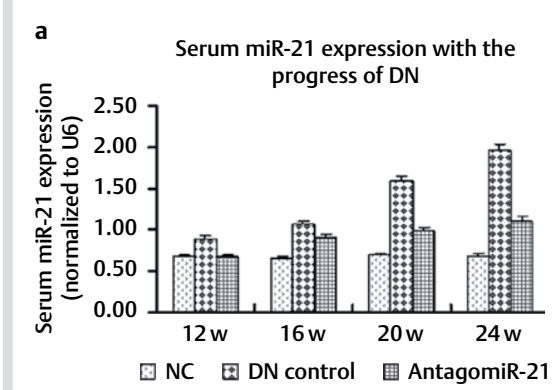

c Alterations of serum miR-21 were consistent with of renal tissue miR-21

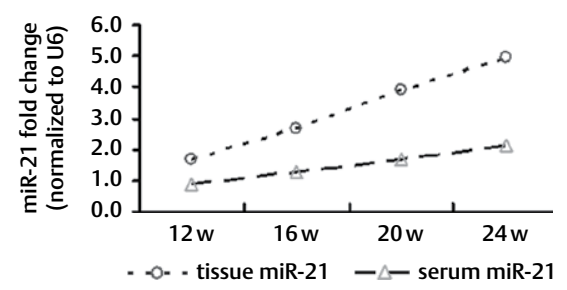

b

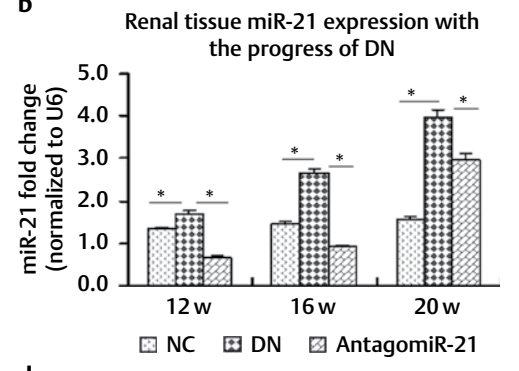

\section{d}

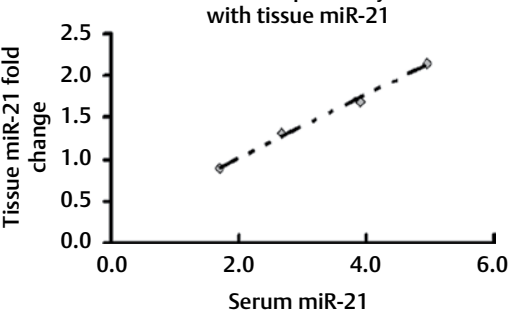

Fig. 1 Relationship between serum miR-21 and tissue miR-21. a Compared with NC group, from 12 to 24 weeks of age, the expression of serum miR-21 was significantly increased in DN group. After the treatment of antagomiR-21, serum miR21 was remarkably decreased $(P<0.05)$. b with the progress of $\mathrm{DN}$, the expression of renal tissue miR-21 was significantly increased in DN group. After the treatment of antagomiR-21, miR-21 expression was remarkably decreased $(P<0.05)$. c The altered trends of renal tissue miR-21 were significantly consistent with serum miR-21. d serum miR-21 was positively correlated with tissue miR-21 expression $(r=0.894, p<0.05)$. 

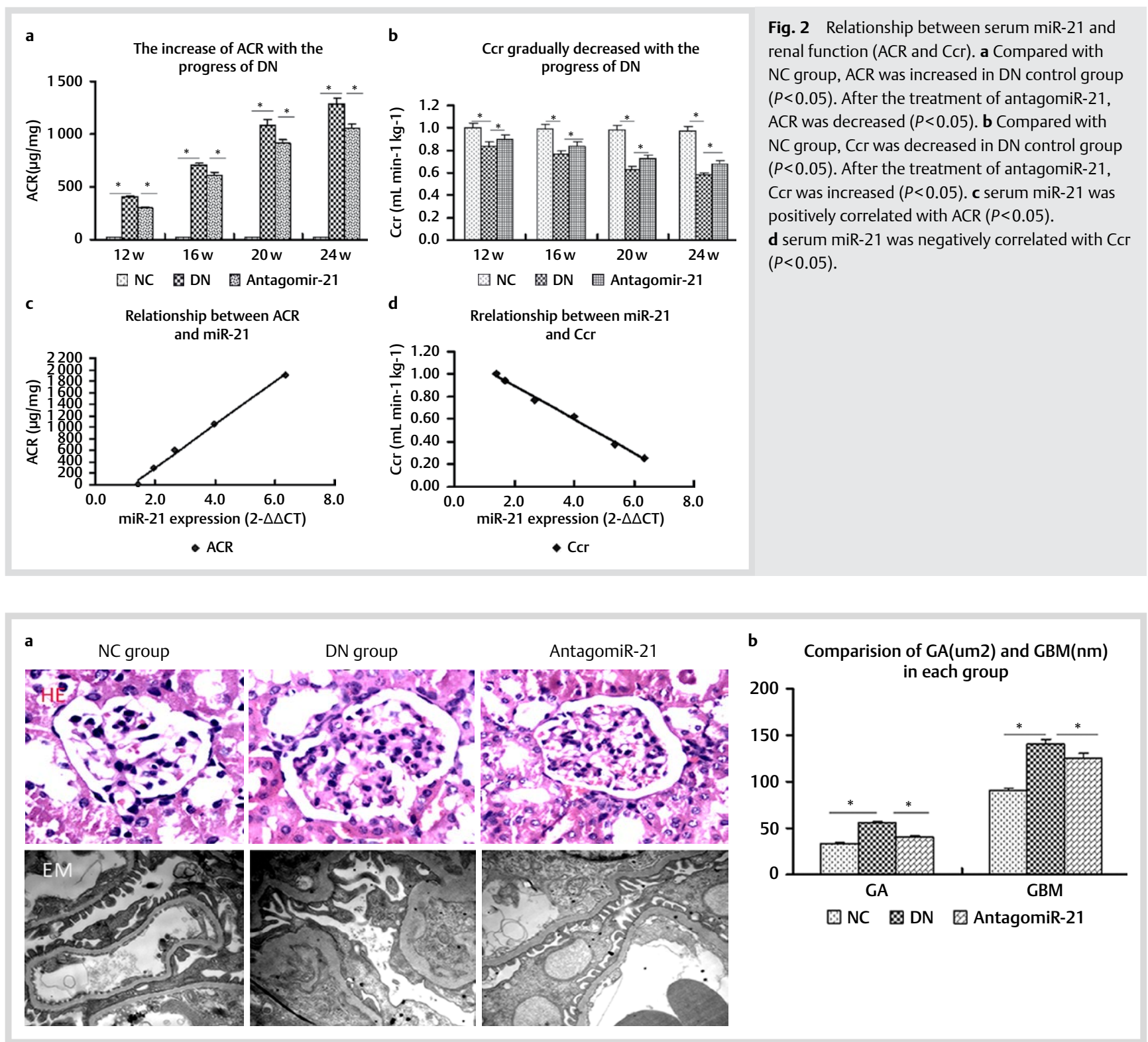

Fig. 3 Morphological changes of renal tissue sections at 24 weeks of age. a GBM thickened and mesangial matrix hyperplasia in DN control group, after the treatment of antagomiR-21, the changes of renal structure were obviously improved. b Compared with NC group, GA and GBM were significantly increased in DN group, after the treatment of antagomiR-21, GA and GBM was slightly but significantly decreased. Light microscopy $\times 400$. Electron microscopy $(E M) \times 20000$. (Color figure available online only).

serum miR-21 may be associated with the morphological changes of DN, whereas, antagomiR-21 ameliorated renal morphology in diabetic kidneys.

\section{Serum miR-21 was closely associated with collagen fibers}

Collagen fibers(CFs) are the most important ingredients comprised of the glomerulus such GBM and mesangial matrix, but the deposition of excessive CFs altered renal structure, and adversely affects renal function [26]. To determine the relationship between serum miR-21 and CFs, we examined CFs by Picrosirius red and Masson staining, respectively. Picrosirius red staining by polarized light microcopy showed that CFs was bright yellow or orange (col-I for red or yellow, col-IV for light yellow ( $\bullet$ Fig. 4a). Similarly, Masson staining showed that a large number of CFs were primarily deposited in GBM, mesangial region and renal interstitial region in DN group compared with
NC group ( $\odot$ Fig. 4a). Next, to discriminate the collagen type, the expression of col-I and col-IV were examined by western blot, we found that col-IV and col-I was remarkably increased in DN group compared with NC group ( $\bullet$ Fig. 4c, d). Interestingly, serum miR-21 was positively correlated with the protein of col-IV rather than correlated with col-I (Pearson correlation, $\left.\mathrm{r}_{\text {col-IV }}=0.870, \mathrm{P}<0.05, \mathrm{r}_{\text {col-I }}=0.39, \mathrm{P}>0.05\right)$. Importantly, after KK-Ay DN mice were injected intraperitoneally with antagomiR-21, the content of collagen fibers (CCF) and col-IV were decreased, instead of col-I. ( $\odot$ Fig. $4 \mathbf{a}-\mathbf{d}$ ). overall, these results suggested serum miR-21 was closely associated with excessive deposition of collagen fibers, especially for col-IV.

\section{miR-21 overexpression decreased target smad7 expression}

As described above, serum miR-21 was closely associated with renal structure and function of DN. then, miR-21 was how to 


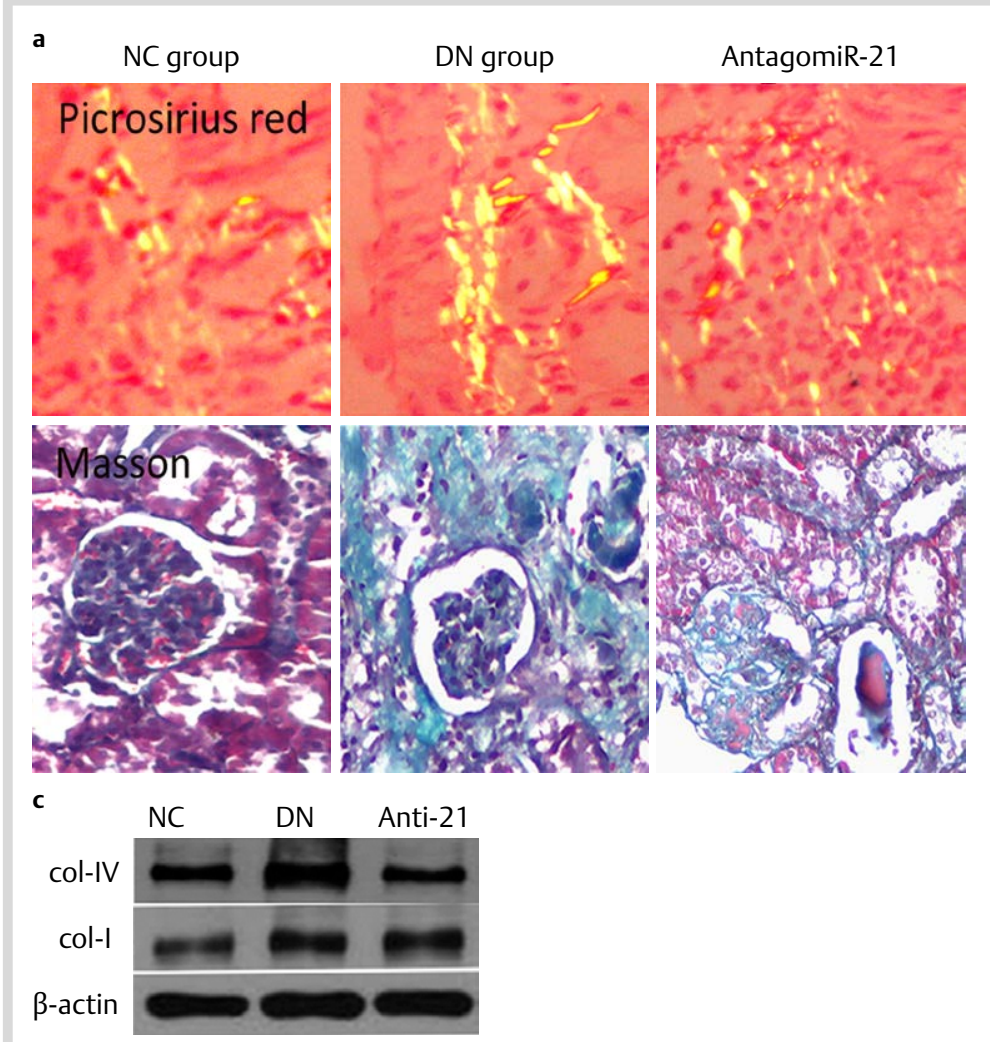

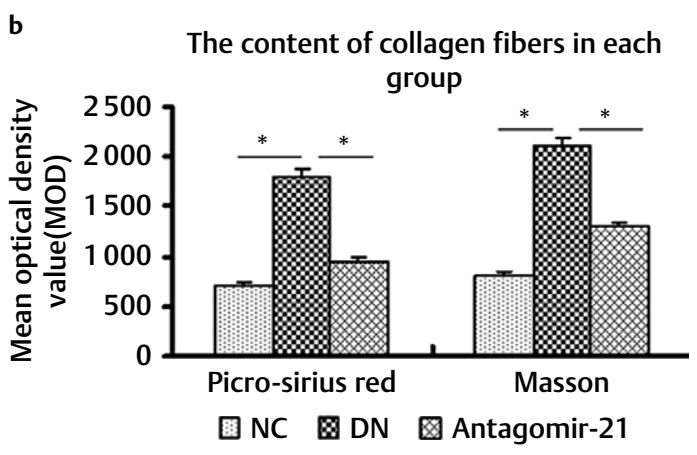

d Quantification of col-IV and col-I western blot

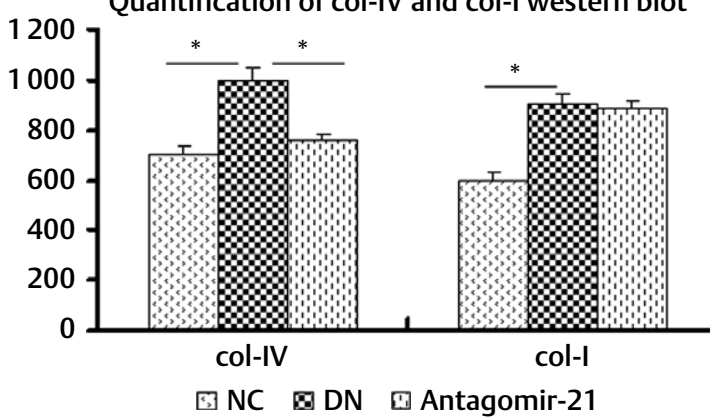

CFs was slightly but significantly decreased. $\mathbf{c}$ Western blotting band of col-IV and col-I protein. d Quantification of col-IV and col-I for western blotting results. The grey value of col-IV and col-I was markedly increased in DN group compared with NC group. After the treatment of antagomiR-21, col-IV was significantly decreased, whereas col-I was not changed. Light microscopy $\times 400$. (Color figure available online only). affect DN. According to TargetScan database (http://www.targetscan.org/), smad7, but not col-IV and col-I, was a potential target of miR-21 ( $\odot$ Fig. 5a). Interestingly, kidney-targeting Smad7 gene transfer may be an effective therapy for type-2 DN, acting via simultaneous modulation of the TGF- $\beta /$ Smads and NF-KB signalling pathways [27]. Smad7 suppresses renal fibrosis via altering expression of TGF- $\beta /$ Smad3-regulated miRNA [28]. Therefore, to further confirm whether smad7 was a validated miR-21 target, we performed the luciferase report gene assays. The results exhibited that wild-type(WT)-luciferase-Smad73'UTR reporter gene for luciferase activity was remarkably decreased compared with mutant type(MT)-luciferase-Smad73'UTR reporter and miR-control plasmid, suggested that smad7 was a validated miR-21 target ( $\odot$ Fig. 5 b, $\mathrm{p}<0.05$ ). Next, to investigate whether miR-21 over-expression and inhibitor on smad7 protein, HKCs were transfected with miR-control, miR-21 overexpression and miR-21 inhibitor lentivirus vector, the results demonstrated that miR-21 over-expression significantly decreased smad7 protein in vitro by Immunofluorescence cell chemistry (ICC) ( $\odot$ Fig. $\mathbf{5 c}, \mathbf{d}, \mathrm{P}<0.05)$. In contrast, miR-21 inhibitor significantly increased smad7 protein ( $\odot$ Fig. $5 c, d, P<0.05$ ). Taken together, our data exhibited that miR-21 was involved in renal injury of DN by directly down-regulating smad7, and that smad7 was a validated miR-21 target.

\section{Discussion \\ $\nabla$}

In recent years, many studies have established many miRs are closely associated with DN $[9,10,20,22]$, mostly focusing on their actions inside the cell from the tissues samples [29]. Because of the presence of potent ribonucleases, most investigators doubted that extracellular RNA could survive in the blood [30]. Up to now, many studies have documented circulating a large number of serum miRs remain stable and consistent in severe conditions [31-34]. It is very possible to detect serum miRs which help to diagnose this disease. Therefore, to indentify the relationship between serum miR-21 and tissue miR-21 expression, we examined serum and tissue miR-21 expression by real-time RT-PCR with the course of DN. We found that serum miR-21 expressions were shown to be increased in KK-Ay DN mice at 12-24 weeks of age. More surprisingly, the altered trends of serum miR-21 levels were significantly consistent with the expressions of renal tissues miR-21. Importantly, antagomiR-21 can decrease the expressions of serum and tissue miR21 . These results suggested that directly detecting serum miR-21 was the best substitute for tissue miR- 21 .

Albumin creatinine cratio (ACR) has been considered as a good clinical predictor of renal lesions in DN [35]. Creatinine clearance ratio (Ccr) is generally considered as marker of renal filtration function [25]. We found that serum miR-21 expression was positively correlated with ACR and negatively correlated with Ccr. Moreover, the change trend of miR-21 was consistent with ACR, suggesting that miR-21 may be a biomarker reflecting for 

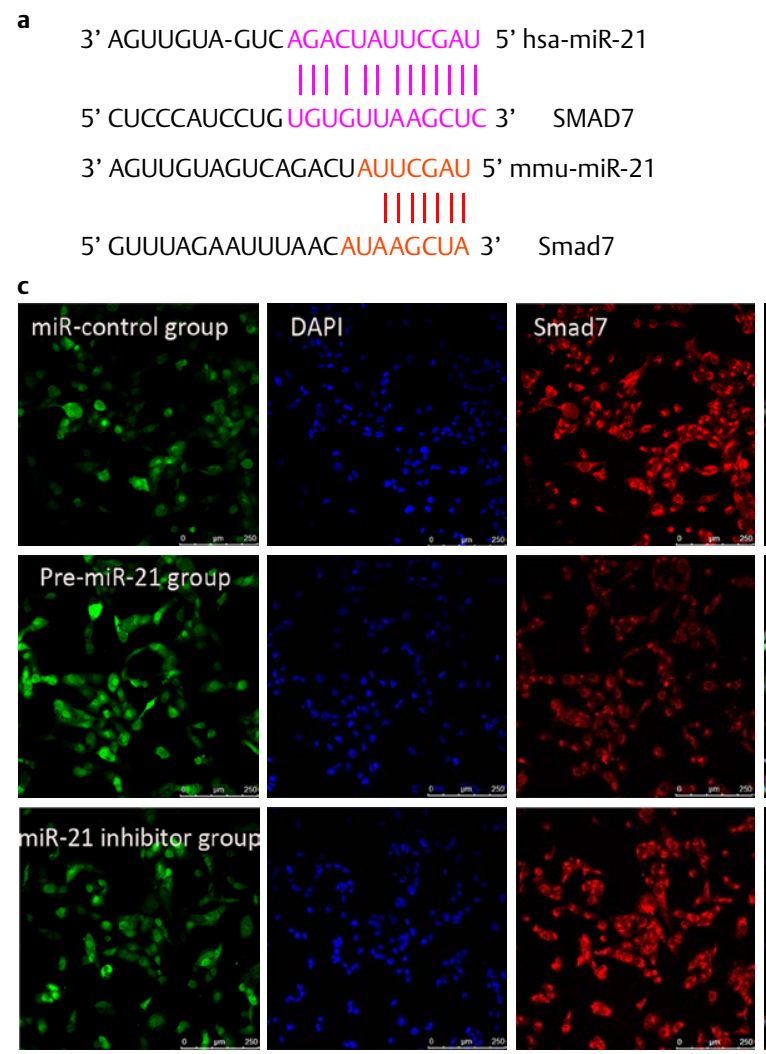
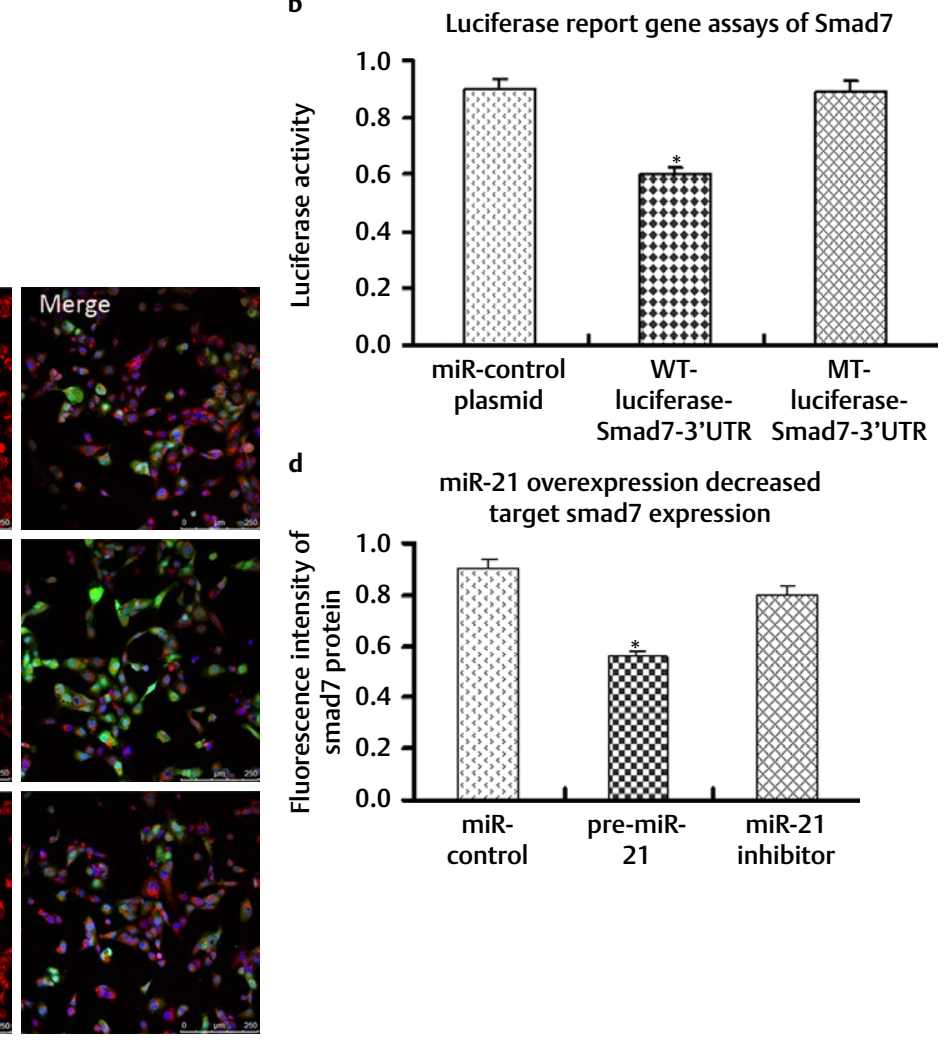

b

Fig. 5 miR-21 overexpression decreased target smad7 expression. a Alignment of hsa-miR-21 and mmu-miR-21 with smad7-3'-UTR based on targetScan software from (http://www.targetscan.org/), several nucleotides in the 5'-region of miR-21 contain a perfect match with the 3'-UTR sequence of smad7 genes. $\mathbf{b}$ The results of luciferase report gene assays. $\mathbf{c}$ Representative photograph of smad7 protein by ICC. $\mathbf{d}$ The fluorescence intensity of smad7 proteins $(p<0.05)$. (Color figure available online only).

the volume of urine protein excretion. More importantly, antagomiR-21 can not only decrease serum miR-21 and ACR but also increase Ccr. Taken together; we concluded that the changes of circulating serum miR-21 can indirectly reflect renal function, which may be as a potential diagnostic biomarker for DN.

A well-organized collagen fiber was necessary to maintain structural and functional integrity of renal tissue. Excessive CFs accumulate in the kidney, which adversely affects the structure of the kidney and further lead to the loss of renal function [36]. Additionally, CFs was the most important ingredients comprised of GBM and mesangial matrix. Moreover, GBM and glomerular area (GA) were sensitive markers of DN [37]. Accumulating studies showed that smad7 may be an effective therapy for DN and renal fibrosis via altering expression of TGF- $\beta 1 /$ Smad3-regulated miRs. Moreover, miR-21 participates in fibrogenic events in kidneys, lungs, heart, or other organs by regulating a unique array of targets $[14,38,39]$. Interestingly, our luciferase report gene assays suggested that smad7 was a validated miR-21 target, miR21 over-expression significantly decreased smad7 expression. All these results showed that miR-21 was involved in the pathogenesis of DN by downregulating target smad7, due to the decrease of smad7, led to the depostion of col-IV and col-I, further resulted in GBM thickened and mesangial matrix hyperplasia. Additionally, we found that serum miR-21 was positively correlated with GBM, GA, CFC and col-IV, whereas, unrelated to col-I. AntgomiR-21 can decrease GBM, GA, CFC and col-IV but not col-I. Thus, we speculated that both circulating serum miR-
21 and tissue local miR-21 were closely associated with CFs formation of DN, especially for col-IV rather than col-I.

In summary, our study suggested that serum miR-21 may be as a potential diagnostic biomarker for $\mathrm{DN}$, both circulating serum and tissue local miR-21 attenuated renal function and structure by inhibiting target smad7. Although our results suggested serum miR-21 may be a new possible marker for the detection of $\mathrm{DN}$, up to now, the measure of urinary albumin excretion rate is the most reliable indicator.

\section{Acknowledgments}

$\nabla$

This study was supported by Grants from the National Natural Science Foundation of China (No. 81560143 and 81560254), Scientific research projects of Health care industry in Gansu province in 2014 (GWGL2014-55 and 2014-59), and the Major National Basic Research Program of China (973 Program, No. 2012CB518602).

Discloures: No conflicts of interest, financial or otherwise, are declared by the authors.

\section{Affiliations}

'Department of Endocrinology, Gansu Provincial People's Hospital, Donggang West Road, Lanzhou, PR China

2 Department of Gynecology and Obstetrics, Gansu Provincial People's

Hospital, Donggang West Road, Lanzhou, PR China 
${ }^{3}$ Clinical College of Ophthalmology, Tianjin Medical University, Tianjin Eye Hospital, Tianjin, China

${ }^{4}$ Metabolic Disease Center, School of Traditional Chinese medical, Capital Medical University, Beijing, China

${ }^{5}$ Department of Endocrinology, Beijing Tongren Hospital, Capital Medical University, Beijing, China

\section{References}

1 Nishi S, Ueno M, Hisaki S et al. Ultrastructural characteristics of diabetic nephropathy. Med Electron Microsc 2000; 33: 65-73 doi:10.1007/ s007950000012

2 Zhong $\mathrm{H}$, Liu F, Fu P et al. A comparison of clinical characteristics and survival between diabetic nephropathy patients and non-diabetic nephropathy patients undergoing peritoneal dialysis. Sichuan Da Xue Xue Bao Yi Xue Ban 2012; 43: 442-446

3 Rychlik I, Miltenberger-Miltenyi G, Ritz E. The drama of the continuous increase in end-stage renal failure in patients with type II diabetes mellitus. Nephrol Dial Transplant 1998; 13 (Suppl 8): 6-10

4 Ito $T$, Tanimoto $M$, Yamada $K$ et al. Glomerular changes in the KK-Ay/ Ta mouse: a possible model for human type 2 diabetic nephropathy. Nephrology 2006; 11: 29-35 doi:10.1111/j.1440-1797.2006.00543.x

5 Perron MP, Provost P. Protein interactions and complexes in human microRNA biogenesis and function. Frontiers in bioscience: a journal and virtual library 2008; 13 : 2537-2547

6 Shantikumar S, Caporali A, Emanueli C. Role of microRNAs in diabetes and its cardiovascular complications. Cardiovasc Res 2012; 93: 583-593 doi:cvr300 [pii]10.1093/cvr/cvr300

7 He A, Zhu L, Gupta N et al. Overexpression of micro ribonucleic acid 29, highly up-regulated in diabetic rats, leads to insulin resistance in 3T3-L1 adipocytes. Mol Endocrinol 2007; 21: 2785-2794 doi:me.2007-0167 [pii]10.1210/me.2007-0167

8 Qin W, Chung AC, Huang XR et al. TGF-beta/Smad3 signaling promotes renal fibrosis by inhibiting miR-29. J Am Soc Nephrol 2011; 22: 14621474 doi:ASN.2010121308 [pii]10.1681/ASN.2010121308

9 Wang $Q$ Wang $Y$, Minto AW et al. MicroRNA-377 is up-regulated and can lead to increased fibronectin production in diabetic nephropathy. FASEB J 2008; 22: 4126-4135 doi:fj.08-112326 [pii]10.1096/ fj.08-112326

10 Zhang Z, Luo X, Ding S et al. MicroRNA-451 regulates p38 MAPK signaling by targeting of Ywhaz and suppresses the mesangial hypertrophy in early diabetic nephropathy. FEBS Lett 2012; 586: 20-26 doi:S00145793(11)00571-0 [pii]10.1016/j.febslet.2011.07.042

11 Caporali A, Meloni M, Vollenkle C et al. Deregulation of microRNA-503 contributes to diabetes mellitus-induced impairment of endothelial function and reparative angiogenesis after limb ischemia. Circulation 2011; 123: 282-291 doi:CIRCULATIONAHA.110.952325 [pii]10.1161/ CIRCULATIONAHA.110.952325

12 Chung AC, Huang XR, Meng X et al. miR-192 mediates TGF-beta/Smad3driven renal fibrosis. Journal of the American Society of Nephrology: JASN 2010; 21: 1317-1325 doi:10.1681/ASN.2010020134

13 Krichevsky AM, Gabriely G. miR-21: a small multi-faceted RNA. Journal of cellular and molecular medicine 2009; 13: 39-53 doi:10.1111/ j.1582-4934.2008.00556.x

14 Zarjou A, Yang S, Abraham E et al. Identification of a microRNA signature in renal fibrosis: role of miR-21. American journal of physiology Renal physiology 2011; 301: F793-F801 doi:10.1152/ajprenal.00273.2011

15 Fiorentino L, Cavalera M, Mavilio $M$ et al. Regulation of TIMP3 in diabetic nephropathy: a role for microRNAs. Acta diabetologica 2013; 50: 965-969 doi:10.1007/s00592-013-0492-8

16 Lai JY, Luo J, O'Connor C et al. MicroRNA-21 in glomerular injury. Journal of the American Society of Nephrology: JASN 2015; 26: 805-816 doi:10.1681/ASN.2013121274

17 Dey N, Das F, Mariappan MM et al. MicroRNA-21 orchestrates high glucose-induced signals to TOR complex 1 , resulting in renal cell pathology in diabetes. The Journal of biological chemistry 2011; 286: 25586-25603 doi:10.1074/jbc.M110.208066

18 Chau BN, Xin C, Hartner J et al. MicroRNA-21 promotes fibrosis of the kidney by silencing metabolic pathways. Science translational medicine 2012; 4: 121 ra118 doi:10.1126/scitranslmed.3003205
19 Zhang Z, Peng H, Chen J et al. MicroRNA-21 protects from mesangial cell proliferation induced by diabetic nephropathy in $\mathrm{db} / \mathrm{db}$ mice. FEBS letters 2009; 583: 2009-2014 doi:10.1016/j.febslet.2009.05.021

20 Zhong X, Chung AC, Chen HY et al. miR-21 is a key therapeutic target for renal injury in a mouse model of type 2 diabetes. Diabetologia 2013; 56: 663-674 doi:10.1007/s00125-012-2804-x

21 Glowacki F, Savary G, Gnemmi $V$ et al. Increased circulating miR-21 levels are associated with kidney fibrosis. PLoS One 2013; 8: e58014 doi:10.1371/journal.pone.0058014PONE-D-12-31316 [pii]

22 Wang J, Gao Y, Ma M et al. Effect of miR-21 on Renal Fibrosis by Regulating MMP-9 and TIMP1 in kk-ay Diabetic Nephropathy Mice. Cell Biochem Biophys 2013; doi:10.1007/s12013-013-9539-2

23 Wang JY, Gao YB, Zhang $N$ et al. miR-21 overexpression enhances TGF-beta1-induced epithelial-to-mesenchymal transition by target smad7 and aggravates renal damage in diabetic nephropathy. Molecular and cellular endocrinology 2014; 392: 163-172 doi:10.1016/j. mce.2014.05.018

24 Krutzfeldt J, Kuwajima S, Braich R et al. Specificity, duplex degradation and subcellular localization of antagomirs. Nucleic Acids Res 2007; 35 : 2885-2892 doi:gkm024 [pii]10.1093/nar/gkm024

25 Yokozawa T, Nakagawa T, Wakaki $\mathrm{K}$ et al. Animal model of diabetic nephropathy. Exp Toxicol Pathol 2001; 53: 359-363

26 Mason RM, Wahab NA. Extracellular matrix metabolism in diabetic nephropathy. J Am Soc Nephrol 2003; 14: 1358-1373

27 Ka SM, Yeh YC, Huang XR et al. Kidney-targeting Smad7 gene transfer inhibits renal TGF-beta/MAD homologue (SMAD) and nuclear factor kappaB (NF-kappaB) signalling pathways, and improves diabetic nephropathy in mice. Diabetologia 2012; 55: 509-519 doi:10.1007| s00125-011-2364-5

28 Chung $A C$, Dong $Y$, Yang $W$ et al. Smad7 suppresses renal fibrosis via altering expression of TGF-beta/Smad3-regulated microRNAs. Molecular therapy: the journal of the American Society of Gene Therapy 2013; 21: 388-398 doi:10.1038/mt.2012.251

29 Meister G. miRNAs get an early start on translational silencing. Cell 2007; 131: 25-28 doi:S0092-8674(07)01209-3 [pii]10.1016/j. cell.2007.09.021

30 El-Hefnawy T, Raja S, Kelly L et al. Characterization of amplifiable, circulating RNA in plasma and its potential as a tool for cancer diagnostics. Clin Chem 2004; 50: 564-573 doi:10.1373/clinchem.2003.0 28506clinchem.2003.028506 [pii]

31 Wang HJ, Zhang PJ, Chen WJ et al. Characterization and Identification of novel serum microRNAs in sepsis patients with different outcomes. Shock 2013; 39: 480-487 doi:10.1097/SHK.0b013e3182940cb8

32 Chen $\mathrm{X}, \mathrm{Ba} Y, \mathrm{Ma}$ L et al. Characterization of microRNAs in serum: a novel class of biomarkers for diagnosis of cancer and other diseases. Cell Res 2008; 18: 997-1006 doi:cr2008282 [pii]10.1038/cr.2008.282

33 Sisco KL. Is RNA in serum bound to nucleoprotein complexes? Clin Chem 2001; 47: 1744-1745

34 Tsui NB, Ng EK, Lo YM. Stability of endogenous and added RNA in blood specimens, serum, and plasma. Clin Chem 2002; 48: 1647-1653

35 Viberti G, Wheeldon NM. Microalbuminuria reduction with valsartan in patients with type 2 diabetes mellitus: a blood pressure-independent effect. Circulation 2002; 106: 672-678

36 Goh SY, Cooper ME. Clinical review: The role of advanced glycation end products in progression and complications of diabetes. J Clin Endocrinol Metab 2008; 93: 1143-1152 doi:jc.2007-1817 [pii]10.1210/ jc.2007-1817

37 Osterby $R$. Morphometric studies of the peripheral glomerular basement membrane. II. Topography of the initial lesions. Diabetologia 1973; 9: 108-114

38 Liu G, Friggeri A, Yang $Y$ et al. miR-21 mediates fibrogenic activation of pulmonary fibroblasts and lung fibrosis. J Exp Med 2010; 207: 1589-1597 doi:jem.20100035 [pii]10.1084/jem.20100035

39 Thum T, Gross C, Fiedler J et al. MicroRNA-21 contributes to myocardial disease by stimulating MAP kinase signalling in fibroblasts. Nature 2008; 456: 980-984 doi:nature07511 [pii]10.1038/nature07511 